\title{
DETERMINING THE REACTION TIME FOR TRIGGERING SUPPORTIVE CONTROL ACTIONS TO GUARANTEE ADEQUATE FREQUENCY RESPONSE IN SMART GRIDS
}

\author{
Jiecai Luo \\ Electrical Engineering Department \\ Southern University and A\&M College \\ 801 Harding Blvd. \\ Baton Rouge, LA 70813, USA \\ luo@subr.edu \\ Mohammed M. Olama \\ Computational Sciences \& Engineering Division \\ Oak Ridge National Laboratory \\ 1 Bethel Valley Rd. \\ Oak Ridge, TN 37831, USA \\ olamahussemm@ornl.gov
}

\author{
Seddik M. Djouadi \\ Min H. Kao Department of Electrical Engineering \\ \& Computer Science \\ University of Tennessee \\ 1520 Middle Drive \\ Knoxville, TN 37996, USA \\ mdjouadi@utk.edu \\ Yichen Zhang \\ Energy Systems Division \\ Argonne National Laboratory \\ 9700 S Cass Ave. \\ Lemont, IL 60439, USA \\ yichen.zhang@anl.gov
}

\begin{abstract}
The progressive integration of renewable energy resources in the modern power grid can result in unsatisfactory frequency responses. To address this problem, wind turbine generators (WTGs), in particular, can be employed to support the power grid. Most works, however, only consider a simple step disturbance. In this paper, a new class of worst-case disturbances is introduced and their nefarious impact on renewable-penetrated power systems is investigated. The worst-case disturbances are derived using optimal control theory. More importantly, the proposed mechanism allows to determine the reaction time to trigger supportive control actions of WTGs to ensure satisfactory frequency response. Numerical results are provided and the effects of the worst-case disturbance on the reaction time are presented.
\end{abstract}

Keywords: smart grids, power system frequency control, optimal control, reaction time, wind turbine generators.

\section{INTRODUCTION}

As the integration of renewable energy resources, such as wind turbines and solar photovoltaic, increases in the power grid around the world, new challenges to the control and operation of power systems are encountered. In particular, the frequency response is impacted when a considerable amount of powerelectronic interfaced generation is connected to the system. The result can be non-satisfactory system frequency response, as a small power disturbance may lead to a large frequency excursion during the 
transient period, that is, the period of inertial and primary responses. This poor transient response can trigger unnecessary over- or under-frequency relay actions, even though the system has adequate capacity to reach a viable steady state (Pulendran and Tate 2017). Thus, maintaining the system frequency within the continuous operational zone, or so-called the safety limits, under a certain set of disturbances has become increasingly important and necessary to real-world power system operations to avoid unnecessary loss of generation and load (Zhang et al. 2017; Zhang 2018; Zhang et al. 2019).

Various frequency supportive functions for wind turbine generators (WTGs) have been proposed in literature. The most common methods are to provide additional signals related to the measured grid frequency deviation (Arani and El-Saadany 2013; Wu and Infield 2013; Wilches-Bernal, Chow, and Sanchez-Gasca 2016; Zhang et al. 2018). Such methods can be referred to as supplementary-signal based methods (Zhang et al. 2017). Other methods mimic the power-angle relation of traditional synchronous generators by means of modifying either the phase-lock loop (PLL) (Zhang et al. 2017; He, Yuan, and Hu 2017; Hu et al. 2017) or the active power controller (Zhang et al. 2018).

Zhang et al. (2017), Zhang (2018), and Zhang et al. (2019) propose a systematic framework of control mode synthesis to ensure adequate frequency response. The framework first derived an optimization formulation to perform reachability analysis. Then, a composite model of the system frequency response (SFR) and WTGs is used as the analytical model for reachability analysis and as the state observer for online control. In these works, only a bounded disturbance is considered. In real engineering practice, however, disturbances are much more sophisticated, leading to complicated dynamical phenomena.

In this paper, the frequency control problem with WTGs under a new class of disturbances is investigated. A new method based on optimal control theory to determine the worst-case disturbance is proposed. More importantly, the method can determine the reaction time to trigger supportive control actions of WTGs to ensure satisfactory frequency response.

The rest of this paper is organized as follows. The system frequency response model incorporating the support response from WTGs is presented in Section 2. The new method based on optimal control theory to determine the reaction time for triggering the support system is developed in Section 3. Simulation results are provided in Section 4 to verify the proposed method and its effects. Finally, the conclusion is presented in Section 5.

\section{PROBLEM STATEMENT}

Following Zhang et al. (2017), consider the SFR model incorporating the support response model of a WTG shown in Figure 1 below.

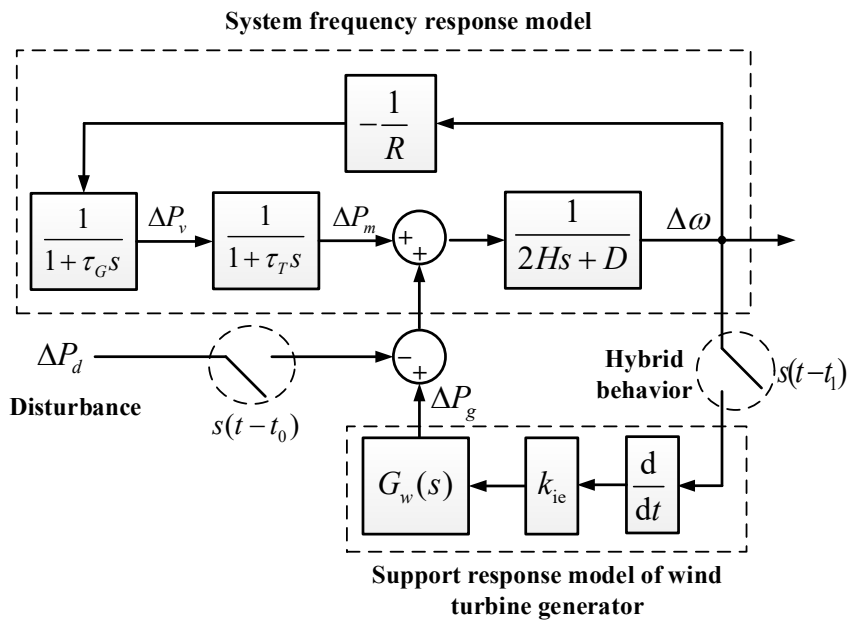

Figure 1: The hybrid system frequency response model incorporating the support response model of a WTG. The supportive mode is limited to the inertia emulation for simplicity (Zhang et al. 2017). 
The shifted unit step function to describe the switching behavior is given as:

$$
s\left(t-t_{k}\right)=\left\{\begin{array}{ll}
1 & t \gg t_{k} \\
0 & \text { otherwise }
\end{array},\right.
$$

and the state-space equation of the SFR is expressed as (Zhang et al. 2017):

$$
\Delta \dot{x}_{s}=A_{s} \Delta x_{s}+B_{s} k_{s c a l} \Delta P_{g}-B_{s} s\left(t-t_{0}\right) \Delta P_{d}
$$

where

$$
\begin{gathered}
A_{S}=\left[\begin{array}{ccc}
-\frac{D}{2 H} & \frac{1}{2 H} & 0 \\
0 & -\frac{1}{\tau_{T}} & \frac{1}{\tau_{T}} \\
-\frac{1}{R \tau_{G}} & 0 & -\frac{1}{\tau_{G}}
\end{array}\right], B_{S}=\left[\begin{array}{c}
\frac{1}{2 H} \\
0 \\
0
\end{array}\right], \\
\Delta x_{S}=\left[\begin{array}{lll}
\Delta \omega & \Delta P_{m} & \Delta P_{v}
\end{array}\right]^{T} .
\end{gathered}
$$

The term $\Delta P_{d}$ denotes the power imbalance due to a disturbance, which is multiplied by the shifted unit step function $s\left(t-t_{0}\right)$ to denote its occurrence instant $t_{0}$, while $\Delta P_{g}$ denotes the output from the WTG associated with the grid supportive controller. The generator speed, mechanical power, valve position, and governor droop are denoted by $\omega, P_{m}, P_{v}$, and $R$, respectively. The terms $H, \tau_{T}$, and $\tau_{G}$ denote inertia constant, turbine time constant, and governor time constant, respectively. The term $k_{\text {scal }}$ denotes a change of base if necessary. Another important piece of this model is to describe the supportive power from the WTG, denoted as the support response model, representing the input-output relation from the measurement signal to the active power variation as shown in the block $G_{w}(s)$ in Figure 1. As described in Zhang et al. (2017), the supportive function is limited to inertia emulation (IE) for simplicity. The support response model of the WTG is governed by the following linear state-space model (Zhang et al. 2017):

$$
\begin{aligned}
\Delta \dot{x_{w}} & =A_{w} \Delta x_{w}+B_{w} s\left(t-t_{1}\right) k_{i e} \Delta \dot{\omega}, \\
\Delta P_{g} & =C_{w} \Delta x_{w}+D_{w} s\left(t-t_{1}\right) k_{i e} \Delta \dot{\omega},
\end{aligned}
$$

where $s\left(t-t_{1}\right)$ denotes the activation signal of the IE mode at $t_{1}$, and $x_{w}$ denotes the state vector of the WTG. A typical example of such a response model can be found in Zhang et al. (2017). The term $k_{\text {ie }}$ is the IE gain. The control objective is described as follows: Consider a computation domain of interest $X \in R^{n}$ within the state space, which can be associated with physical system limits (Zhang et al. 2017). Assume a power imbalance occurs at time $t_{0}$. Given the IE mode with $k_{\text {ie }}$, the objective of the control is to activate the WTG supportive mode at time $t_{1}=t_{0}+t_{r}$ so that the frequency response is adequate, i.e. $\omega \in X_{S}=$ $\left\{\omega_{\text {lim }}^{-} \leq \omega \leq \omega_{\text {lim }}^{+}\right\} \cap X$. The set $X_{S}$ is usually denoted as the safe set, and its complementary set is called the unsafe set $X_{U}=\left\{\omega>\omega_{\text {lim }}^{+}\right.$or $\left.\omega<\omega_{\text {lim }}^{-}\right\} \cap X$. The frequency safety limits are usually defined for a set of contingencies, i.e., $\Delta P_{d} \in D=\left\{\delta \mid \delta_{\text {lim }}^{-} \leq \delta \leq \delta_{\text {lim }}^{+}\right\}$(Zhang et al. 2017; Zhang 2018; Zhang et al. 2019). And the most important task is to determine the reaction time $t_{r}$.

So far, there are few methods developed yet to compute the reachable set caused by the disturbance for a stable system even for linear time-invariant systems. This issue is addressed by developing a new method in Section 3. Before doing so, here it is assumed that in the normal ideal case, the power system (represented by Eqs. (1)-(3)) is stable, and when there is no disturbance $\left(\Delta P_{d}=0\right)$, then $\Delta x_{s}=\left[\begin{array}{lll}\Delta \omega & \Delta P_{m} & \Delta P_{v}\end{array}\right]^{T}=$ $\left[\begin{array}{lll}0 & 0 & 0\end{array}\right]^{T}$, and $\Delta P_{g}=0$. Without loss of generality, assume the initial time is $t_{0}=0$.

The safety set $X_{S}$ is characterized by the disturbance bound, that is $\omega \in X_{S}$, if $|e| \leq k_{1}$, no support system control action is needed. However, when the condition $|e| \leq k_{1}$ is violated, i.e., $\omega \notin X_{S}$, then the worst- 
case disturbance that maximally shortens the reaction time $t_{r}$ is determined based on optimal control theory as presented in the next section.

\section{A NEW METHOD TO ESTIMATE THE REACTION TIME}

Nowadays, multiple grid supportive functionalities have been integrated into the power electronics converters, which make them multi-mode devices. Increasing penetration of such devices makes the power system become a hybrid system, where different continuous dynamics are activated under the pre-defined control logics. Therefore, the reaction time estimation is crucial for the control logic design of such hybrid systems. In this section, we seek to construct the worst-case stressing disturbances to test the robustness of the IE system. We consider a class of disturbances that will force the system into a limit cycle oscillation (Lee and Luo 1998). In general, power systems exhibit stable and unstable limit cycles, in particular when they are subject to heavy loads. Specifically, power systems are susceptible to Hopf bifurcations and the consequent oscillatory instabilities as described in Reddy and Hiskens (2005) and Howell and Venkatasubramanian (1999). For example, Reddy and Hiskens (2005) provide a situation where generator field-voltage limits capture the dynamical behavior of power systems, giving rise to a stable limit cycle. Howell and Venkatasubramanian (1999) use Hopf bifurcation theory to show that unstable equilibrium points are likely to be present in the transient stability boundary when the operating condition has poorly damped oscillatory modes, which are subcritical in power systems. Chiang and Alberto (2015) provide a general reference where these problems are discussed in detail.

The worst-case disturbance considered in this paper is based on using the time distance from the equilibrium as a measure of disturbance severity. In this case, the worst-case disturbance is the one that maximizes the time distance from the origin as an equilibrium point. This strategy is a delay tactic. i.e., it results in a maximized delay (Lee and Luo 1997; Lee and Luo 1997; Lee and Luo 2000; Luo and Lee 2000).

The maximum time distance index is based on the reachable sets from the origin (equilibrium point), the boundary of which provides a nested set of time marks called the isochrones (Lee and Luo 1998). Constructing a sequence of safety isochrones for $|e|=\bar{k}, \bar{k}>k_{1}$, (with no support control action that is $\left.|e|=\left|\Delta P_{d}\right|=k_{1}\right)$ until the last isochrone reaches the unsafe region $X_{U}$.

According to Lee and Luo (1997), Lee and Luo (1997), Lee and Luo (2000), and Luo and Lee (2000), the procedure is as follows: Consider the general nonlinear model as given by the ordinary differential equation in state space form for the controlled system:

$$
\begin{array}{cl}
\dot{x}(t)=f(x(t))+b v(t),|v(t)| \leq \alpha, & x\left(t_{0}\right)=x_{0}, \\
y(t)=g(x(t)), & f(0)=0 .
\end{array}
$$

It is assumed that $x=0$ is an asymptotically stable equilibrium point of $\dot{x}(t)=f(x(t))$, and that $f(x), g(x) \in C^{1}$ (continuously differentiable). The delay tactic objective is to choose the bounded disturbance $v(t)$ to maximize the performance index:

$$
J=\max _{v} \int_{t_{0}}^{t_{f}} d t=\max _{v}\left(t_{f}-t_{0}\right),
$$

where the free final time $t_{f}=t_{f}(v)$ is subject to the end point constraints and $|v(t)| \leq \alpha$. The delay tactics correspond to shortening the support system response time as much as possible.

To solve for $J$, define the Hamiltonian as $H:=1+\eta^{T}(f(x)+b v(t))$, then maximize $H$ with respect to $|v(t)| \leq \alpha$ to get the worst-case disturbance $v^{*}(t)=\alpha \operatorname{sign}\left(\eta^{T} b\right)$, where $\operatorname{sign}(\cdot)$ denotes the sign 
function. This leads to the following worst-case system (Lee and Luo 1997; Lee and Luo 1997; Lee and Luo 2000; Luo and Lee 2000):

$$
\begin{gathered}
\dot{x}(t)=f(x)+b \alpha \operatorname{sign}\left(\eta^{T} b\right), \quad x\left(t_{0}\right)=x_{0}, \\
\dot{\eta}=-\left(\frac{\partial H}{\partial x}\right)^{T}=-\left(\frac{\partial f}{\partial x}\right)^{T} \eta(t),
\end{gathered}
$$

Because the initial data of $\eta\left(t_{0}\right)$ is to be decided, thus the isochronal function $T(x)$ and switch-curve function can be used in the synthesis of the worst-case disturbance $v^{*}(t)$. Since $\eta\left(t_{0}\right)$ is any vector with $\left\|\eta\left(t_{0}\right)\right\|=1$, the subsequent worst-case disturbance can be written as $\max _{\eta\left(t_{0}\right)}\{J\}$, where $v^{*}(t)=$ $\alpha \operatorname{sign}\left(\eta^{T} b\right)$ according to the system in Eq. (5) (Lee and Luo 1997; Lee and Luo 1997; Lee and Luo 2000; Luo and Lee 2000).

Here, $v(t)$ corresponds to $\Delta P_{d}$ and $\alpha$ to $\bar{k}$. Note that the proposed safety isochrones method is based on the worst-case scenario. If we only consider the disturbance to be of the form $\Delta P_{d}=k$, where $k$ is a positive constant parameter, the corresponding safety region will be larger than the one for the worst-case scenario.

It will be shown that the larger $\bar{k}$ is, the smaller $t_{r}$ will be, where $t_{r}$ is the response time (after a disturbance occurs) when the support system is triggered. In the original support system, the linear state-space model in Eq. (3) can be written as:

$$
\begin{gathered}
\Delta \dot{x_{w}}=A_{w} \Delta x_{w}+\left[B_{w} \Delta \omega+(P+I / s)\left(\Delta P_{d}-k_{s c a l} \Delta P_{g}\right)\right] \\
\Delta P_{g}=C_{w} \Delta x_{w}
\end{gathered}
$$

where $P$ and $I$ are constant parameters to be chosen to guarantee the stability of the system.

By the discussion above, it takes a while for $k_{s c a l} \Delta P_{g}$ to follow $\Delta P_{d}$, and $\Delta \omega \rightarrow 0$. This means that $|e|=\bar{k}$ is put back in the condition $|e| \leq k_{1}$, which guarantees the whole system is working inside the safety region. And if $\left|\Delta P_{d}\right|<k_{1}$, then the WTG support system can be released to its normal mode again as shown in Figure 2 .

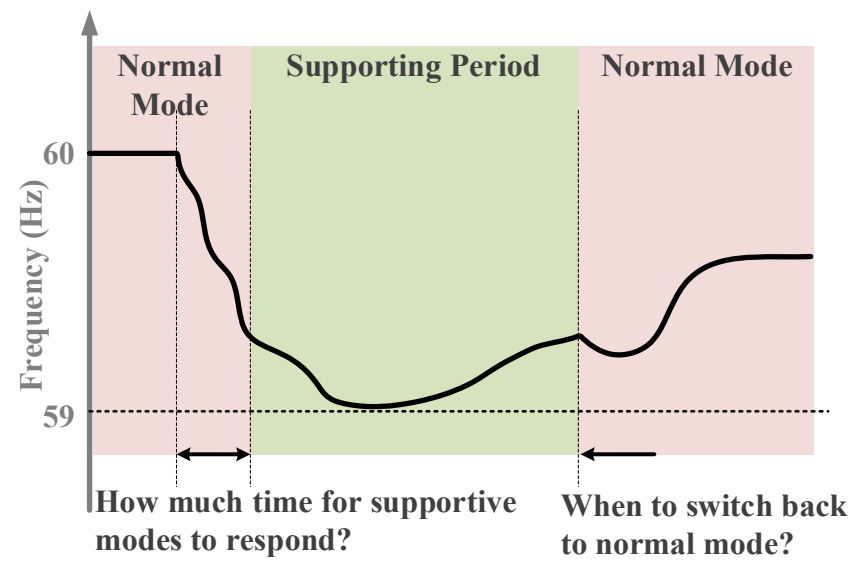

Figure 2: Challenges of synthesizing the supportive modes as the switching instants between modes to achieve an adequate frequency response (Zhang et al. 2017).

According to Zhang et al. (2017), Zhang (2018), Gholami, Shekari, and Sun (2018), and Zhang et al. (2019), the transfer function of the SFR model can be written as:

$$
H(s)=\frac{\alpha_{1} s^{2}+\alpha_{2} s+\alpha_{3}}{s^{3}+\beta_{1} s^{2}+\beta_{2} s+\beta_{3}},
$$


where

$$
\begin{gathered}
\alpha_{1}=\frac{1}{2 H}, \alpha_{2}=\frac{1}{2 H}\left(\frac{1}{\tau_{T}}+\frac{1}{\tau_{G}}\right), \alpha_{3}=\frac{1}{2 H \tau_{T} \tau_{G}} \\
\beta_{1}=\frac{\bar{D}}{2 H}+\frac{1}{\tau_{T}}+\frac{1}{\tau_{G}}, \beta_{2}=\frac{1}{\tau_{T} \tau_{G}}+\frac{\bar{D}}{2 H}\left(\frac{1}{\tau_{T}}+\frac{1}{\tau_{G}}\right), \beta_{3}=\frac{\frac{1}{R}+\bar{D}}{2 H \tau_{T} \tau_{G}} .
\end{gathered}
$$

The state-space model is described in Eqs. (2)-(3), where the input (as discussed above) is $e=s\left(t-t_{r}\right) k_{s c a l} \Delta P_{g}-\Delta P_{d}$, and the output is $\Delta \omega$. Then, the transfer function in Eq. (7) can be realized as:

$$
\begin{gathered}
\frac{\Delta \omega(s)}{e(s)}=H(s)=\frac{\alpha_{1} s^{2}+\alpha_{2} s+\alpha_{3}}{s^{3}+\beta_{1} s^{2}+\beta_{2} s+\beta_{3}}, \\
X(t)=\left[\begin{array}{l}
x_{1}(t) \\
x_{2}(t) \\
x_{3}(t)
\end{array}\right]=\left[\begin{array}{l}
x(t) \\
\dot{x}(t) \\
\ddot{x}(t)
\end{array}\right]=>\dot{X}(t)=A X(t)+b e(t), \\
A=\left[\begin{array}{ccc}
0 & 1 & 0 \\
0 & 0 & 1 \\
-\beta_{3} & -\beta_{2} & -\beta_{1}
\end{array}\right], b=\left[\begin{array}{l}
0 \\
0 \\
1
\end{array}\right] \\
\Delta \omega(t)=C X(t), C=\left[\begin{array}{lll}
\alpha_{3} & \alpha_{2} & \alpha_{1}
\end{array}\right] .
\end{gathered}
$$

The parameters for the simulation are taken from Zhang et al. (2017) as: $H=2(s), R=\frac{1}{30}, \tau_{G}=$ $0.1(s), \tau_{T}=0.5(s)$, and $\bar{D}=1$. So, the transfer function becomes

$$
H(s)=\frac{0.25 s^{2}+3 s+5}{s^{3}+12.25 s^{2}+23 s+155}
$$

which for simplicity is further reduced to a $2^{\text {nd }}$ order model as in Zhang et al. (2017) as:

$$
H_{d}(s)=\frac{0.2414 s+0.4382}{s^{2}+0.8256 s+13.57} \text {. }
$$

The numerical results are based on the reduced order model $H_{d}(s)$ and presented in the next section.

\section{SIMULATION RESULTS}

The $2^{\text {nd }}$ order transfer function $H_{d}(s)$ presented in Section 3 can be realized by the following $2^{\text {nd }}$ order statespace model:

$$
\begin{gathered}
\dot{X}(t)=A X(t)+b e(t), A=\left[\begin{array}{cc}
0 & 1 \\
-13.57 & -0.8256
\end{array}\right], b=\left[\begin{array}{l}
0 \\
1
\end{array}\right], \\
\Delta \omega(t)=C X(t), C=\left[\begin{array}{ll}
0.4382 & 0.2414
\end{array}\right],|e(t)| \leq \alpha .
\end{gathered}
$$

And according to Eq. (5), the worst-case system is

$$
\begin{gathered}
\dot{X}(t)=A X(t)+b \alpha \operatorname{sign}\left(\eta_{2}(t)\right), \\
\dot{\eta}=-\left(\frac{\partial H}{\partial x}\right)^{T}=-(A)^{T} \eta(t), \text { and }
\end{gathered}
$$




$$
\eta(t)=\left[\begin{array}{l}
\eta_{1}(t) \\
\eta_{2}(t)
\end{array}\right], e^{*}(t)=\alpha \operatorname{sign}\left(\eta_{2}(t)\right)
$$

The corresponding numerical results are provided in Figures 3-10. Figures 3-5 show the isochrones at different frequency deviation bounds: $0.2,0.4$, and 0.6 for the worst-case disturbances with $\left|\Delta P_{d}\right| \leq 2.5$. Figures 6-10 show how the response time $t_{r}$ varies as a function of the disturbance upper bound for increasing values of the frequency bound. Depending on the latter, under a certain disturbance threshold, no support action is required. However, as expected the larger the disturbance bound is, the shorter the reaction time will be. In contrast, the larger the frequency bound is, the larger the reaction time will be. Figures 3-10 capture precisely these variations.

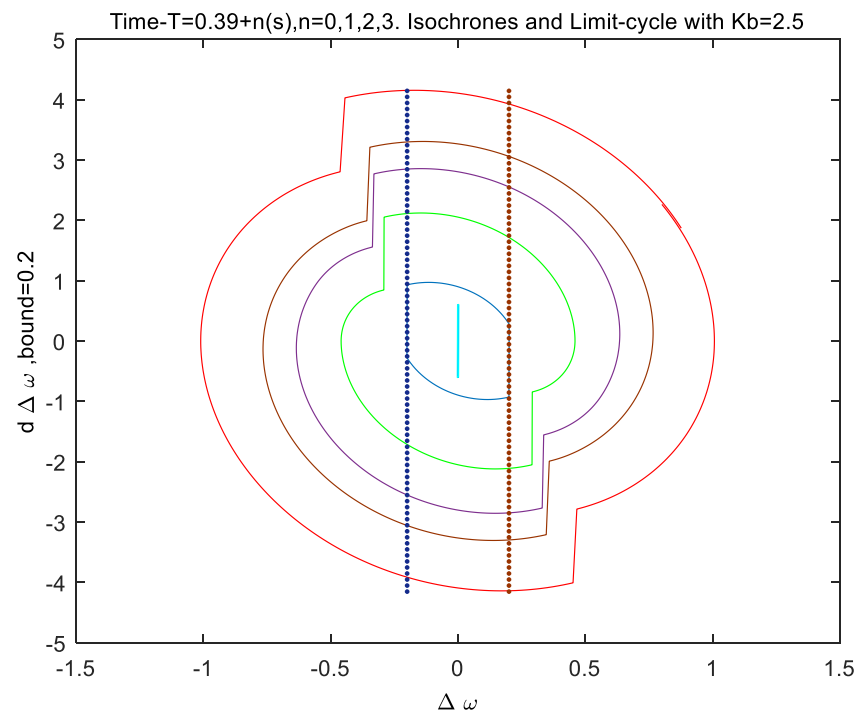

Figure 3: Isochrones for $T=0.39 \mathrm{~s}, 1.39 \mathrm{~s}, 2.39 \mathrm{~s}, 3.39 \mathrm{~s}$ and limit-cycles with $\max \left|\Delta P_{d}\right|=2.5$, frequency bound $=0.2$.

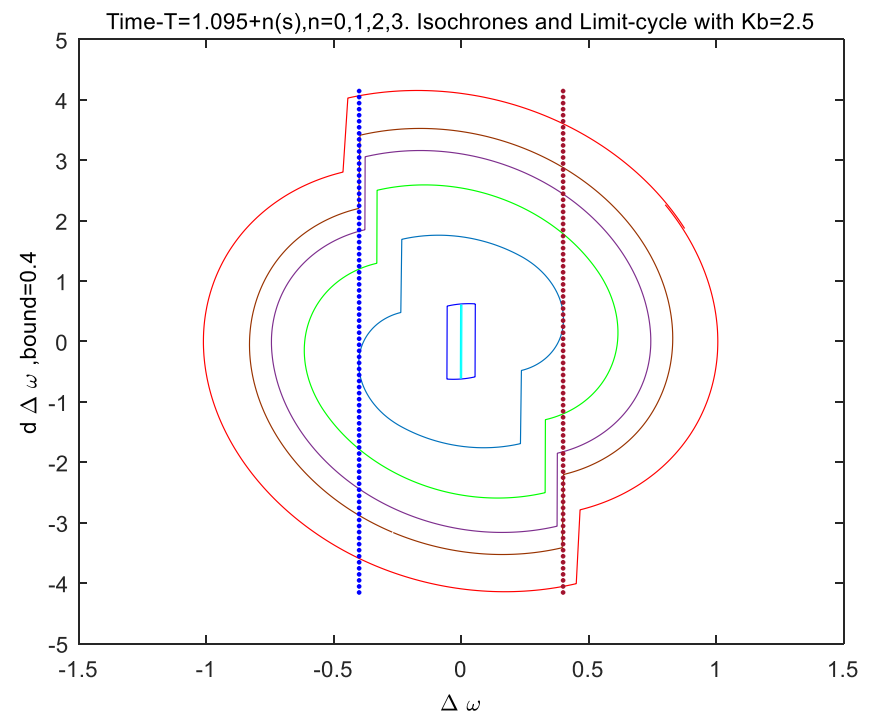

Figure 4: Isochrones for $T=1.095 \mathrm{~s}, 2.095 \mathrm{~s}, 3.095 \mathrm{~s}, 4.095 \mathrm{~s}$ and limit-cycles with $\max \left|\Delta P_{d}\right|=2.5$, frequency bound $=0.4$. 
For the reduced $2^{\text {nd }}$ order model, its reaction time $t_{r}$ to the magnitudes of worst-case disturbances are illustrated (for different frequency error bounds: 0.3, 0.4, 0.5, 0.6 and 0.7) in Figures 6-10. For example, from Figure 10, if the frequency bound equals to $0.7, \max \left|\Delta P_{d}\right|<1.7$, no support action system should be added on to the original system. Once the worst case disturbance $\max \left|\Delta P_{d}\right|>1.7$, say $\max \left|\Delta P_{d}\right|=3$, the reaction time $t_{r}$ is approximately three seconds, this means the support action system should be triggered no later than three seconds, otherwise the system frequency will reach the bound 0.7 .

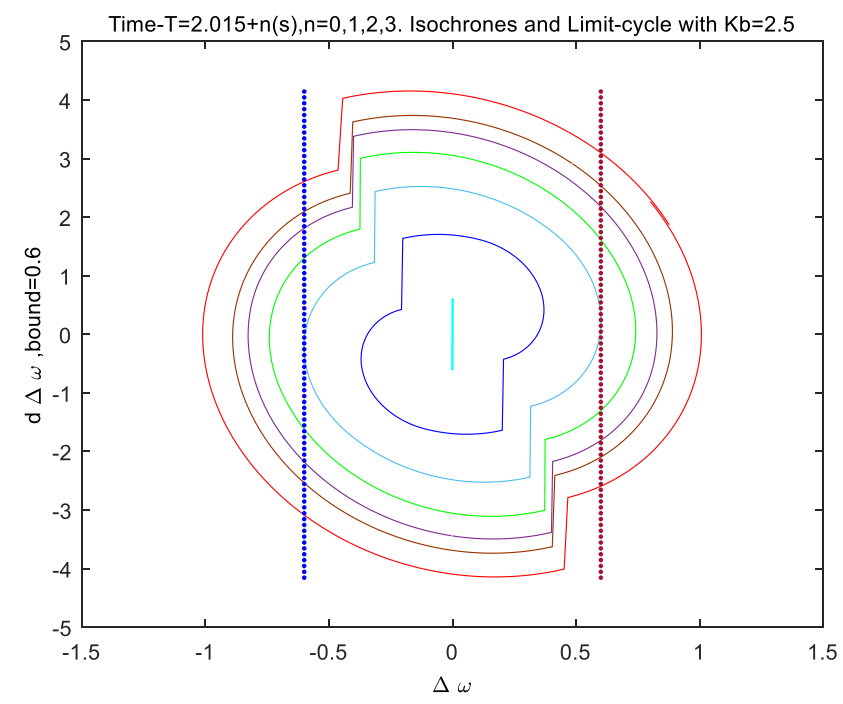

Figure 5: Isochrones for $T=2.015 \mathrm{~s}, 3.015,4.015 \mathrm{~s}, 5.015 \mathrm{~s}$ and limit-cycles with $\max \left|\Delta P_{d}\right|=2.5$, frequency bound $=0.6$.

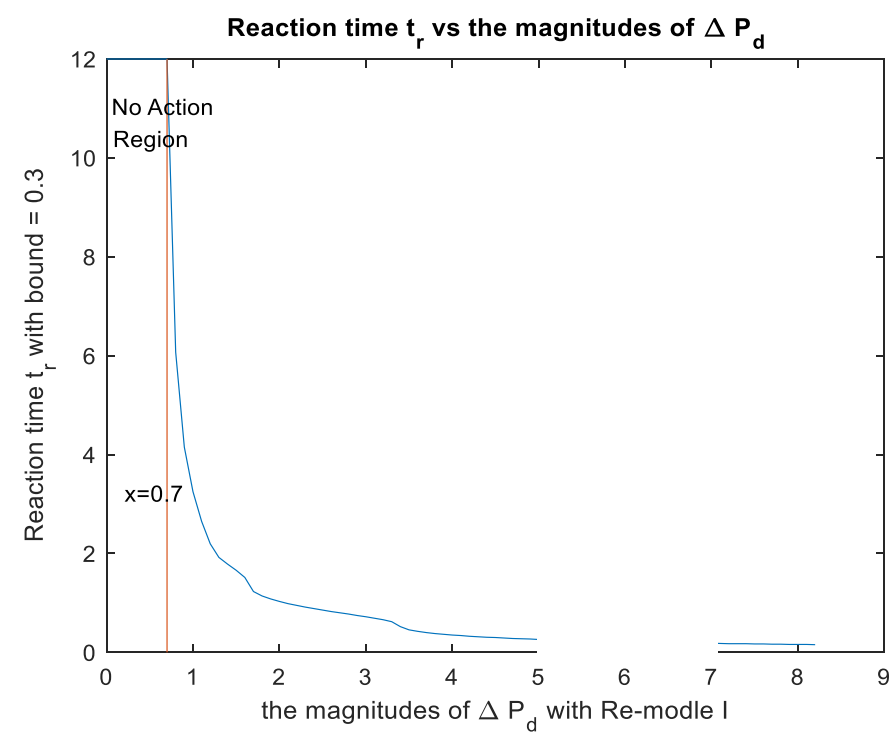

Figure 6: Reaction time $t_{r}$ vs $k_{b}\left(\max \left|\Delta P_{d}\right|\right)$ with frequency bound $=0.3$. 
Luo, Djouadi, Olama, and Zhang

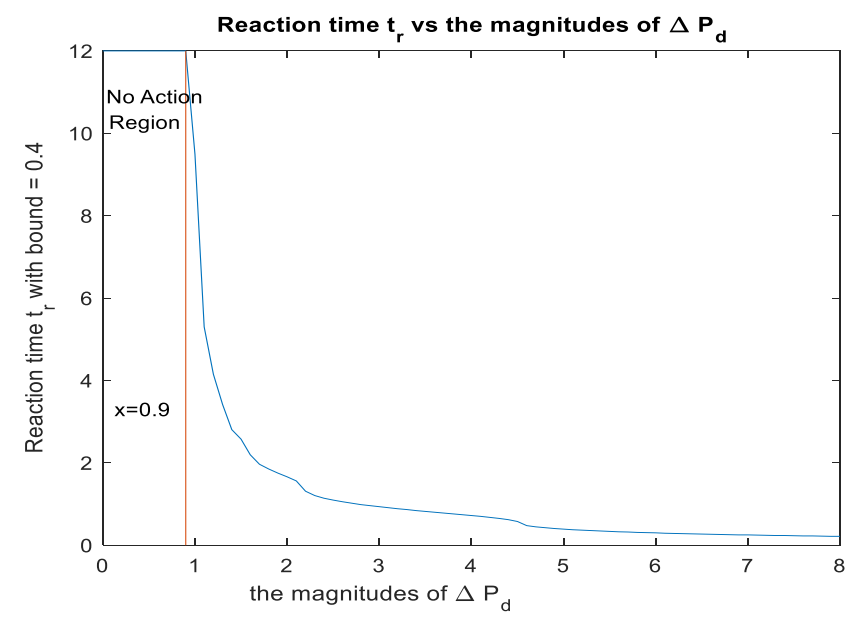

Figure 7: Reaction time $t_{r}$ vs $k_{b}\left(\max \left|\Delta P_{d}\right|\right)$ with frequency bound $=0.4$.

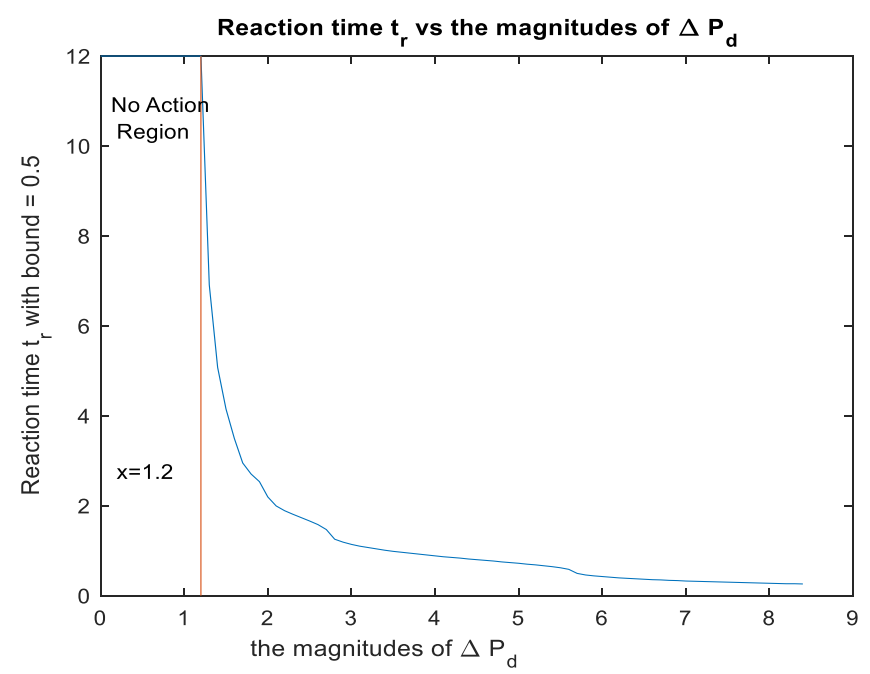

Figure 8: Reaction time $t_{r}$ vs $k_{b}\left(\max \left|\Delta P_{d}\right|\right)$ with frequency bound $=0.5$.

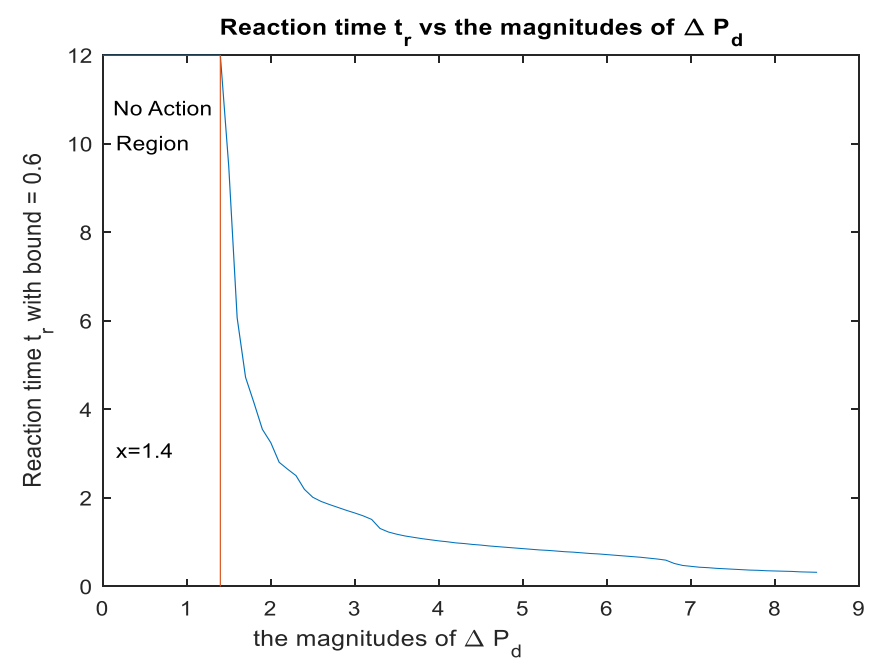

Figure 9: Reaction time $t_{r}$ vs $k_{b}\left(\max \left|\Delta P_{d}\right|\right)$ with frequency bound $=0.6$. 


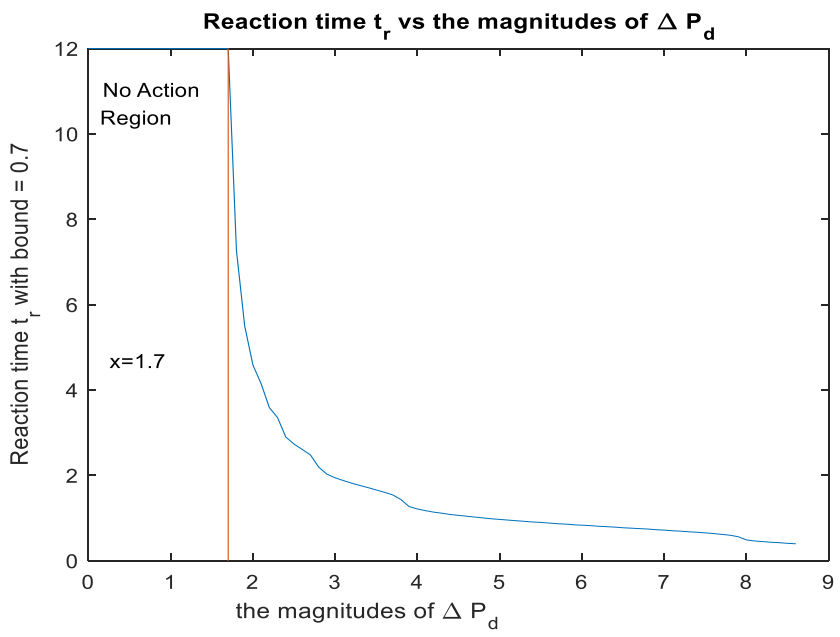

Figure 10: Reaction time $t_{r}$ vs $k_{b}\left(\max \left|\Delta P_{d}\right|\right)$ with frequency bound $=0.7$.

\section{CONCLUSION}

A new class of disturbances on renewable-penetrated power systems and its worst-case derivation is investigated. In addition, a new method to determine the reaction time for triggering support system for WTGs is developed. The proposed mechanism relies on optimal control theory, where the worst-case disturbance is computed based on maximizing a time performance index. The latter corresponds to the maximum distance degrading index based on the reachable sets from the equilibrium. The effectiveness of the proposed mechanism is illustrated by numerical results.

\section{ACKNOWLEDGMENTS}

Research sponsored by the Laboratory Directed Research and Development Program of Oak Ridge National Laboratory (ORNL), managed by UT-Battelle, LLC for the U.S. Department of Energy under Contract No. DE-AC05-00OR22725. In addition, this research has been supported in part by NSF-EPCN award \# 1711432, and a Joint Directed Research and Development (JDRD) Program award.

This manuscript has been authored by UT-Battelle, LLC under Contract No. DE-AC05-00OR22725 with the U.S. Department of Energy. The United States Government retains and the publisher, by accepting the article for publication, acknowledges that the United States Government retains a non-exclusive, paid-up, irrevocable, world-wide license to publish or reproduce the published form of this manuscript, or allow others to do so, for United States Government purposes. The Department of Energy will provide public access to these results of federally sponsored research in accordance with the DOE Public Access Plan (http://energy.gov/downloads/doe-public-access-plan).

\section{REFERENCES}

Arani, M. F. M., and E. F. El-Saadany. 2013. "Implementing virtual inertia in DFIG-based wind power generation”. IEEE Transactions on Power Systems vol. 28 (2), pp. 1373-1384.

Chiang, H.-D., and L. F. C. Alberto, 2015. Stability Regions of Nonlinear Dynamical Systems: Theory, Estimation, and Applications. Cambridge University Press.

Gholami, A., T. Shekari, and X. A. Sun. 2018. "An optimal optimization-based load shedding scheme in microgrids". In Proceedings of the $51^{\text {st }}$ Hawaii International Conference on System Sciences, pp. 26602669. 
He, W., X. Yuan, and J. Hu. 2017. "Inertia provision and estimation of PLL-based DFIG wind turbines," IEEE Transactions on Power Systems vol. 32 (1), pp. 510-521.

Howell, F., and V. Venkatasubramanian. 1999. "Transient stability assessment with unstable limit cycle approximation”. IEEE Transactions on Power Systems vol. 14 (2), pp. 667-677.

Hu, J., S. Wang, W. Tang, and X. Xiong. 2017. "Full-capacity wind turbine with inertial support by adjusting phase-locked loop response". IET Renewable Power Generation vol. 11 (1), pp. 44-53.

Lee, E. B., and J. Luo. 1997. "Two methods for constructing aggrandized disturbances using delay tactics for stabilized control systems". In Proceedings of the American Control Conference (ACC), pp. 21892192.

Lee, E. B., and J. Luo. 1998. "Disturbance stress for stabilized control systems". Mathematical Models and Methods in Applied Sciences vol. 8 (7), pp. 1155-1182.

Lee, E. B., and J. Luo. 2000. "A closed analytic form for the time maximum disturbance isochrones of second-order linear systems". Systems \& Control Letters vol. 40, pp. 229-245.

Luo, J., and E. B. Lee. 2000. "On evaluating the bounded input bounded output stability integral for secondorder systems". IEEE Transactions on Automatic Control vol. 45, pp. 311-312.

Pulendran, S., and J. E. Tate. 2017. "Energy storage system control for prevention of transient underfrequency load shedding". IEEE Transactions on Smart Grid vol. 8 (2), pp. 927-936.

Reddy, P. B., and I. A. Hiskens. 2005. "Limit-induced stable limit cycles in power systems". In Proceedings of the IEEE Russia Power Tech, pp. 1-5.

Wilches-Bernal, F., J. H. Chow, and J. J. Sanchez-Gasca. 2016. "A fundamental study of applying wind turbines for power system frequency control”. IEEE Transactions on Power Systems vol. 31 (2), pp. $1496-1505$.

Wu, L., and D. G. Infield. 2013. "Towards an assessment of power system frequency support from wind plant-modeling aggregate inertial response”. IEEE Transactions on Power Systems vol. 28 (3), pp. 2283-2291.

Zhang, Y. 2018. "Utilizing converter-interfaced sources for frequency control with guaranteed performance in power systems". Ph.D. Dissertation, University of Tennessee, Knoxville, TN, USA.

Zhang, Y., A. Melin, S. M. Djouadi, M. M. Olama, and K. Tomsovic. 2018. "Provision for guaranteed inertial response in diesel-wind systems via model reference control". IEEE Transactions on Power Systems vol. 33 (6), pp. 6557-6568.

Zhang, Y., M. Olama, A. Melin, Y. Xue, S. Djouadi, and K. Tomsovic. 2018. "Synthesizing distributed energy resources in microgrids with temporal logic specifications". In Proceedings of the $9^{\text {th }}$ IEEE International Symposium on Power Electronics for Distributed Generation Systems (PEDG), pp. 1-7.

Zhang, Y., M. Raoufat, K. Tomsovic, and S. M. Djouadi. 2019. "Set theory-based safety supervisory control for wind turbines to ensure adequate frequency response". IEEE Transactions on Power Systems vol. 34 (1), pp. 680-692.

Zhang, Y., K. Tomsovic, S. Djouadi, and H. Pulgar-Painemal. 2017. "Hybrid controller for wind turbine generators to ensure adequate frequency response in power networks". IEEE Journal on Emerging and Selected Topics in Circuits and Systems vol. 7 (3), pp. 359-370.

\section{AUTHOR BIOGRAPHIES}

JIECAI LUO received his Ph.D. from the University of Minnesota in 1999, and currently serves as a Full Professor of Electrical Engineering at Southern University Baton Rouge. His research interest includes signal and image processing, data fusion, power systems, and optimal control systems. Dr. Luo has conducted or is conducting research in several areas: "DSP-based Motion Controls and their Advanced Control Algorithms Study" with Raytheon; and "Advanced Image Processing Techniques for Maximum 
Information Recovery" with the AFRL at WPAFB; "Detection and Sensing of Environmental and Chemical Substances using Ad-hoc Wireless" with DoE; "Data compression effect on (RF) Signal Geolocation" with the AFRL at Wright Patterson AFB. "Switched Mode Controls for Guaranteeing Adequate Frequency Response of the Smart Grid' with ORNL 2019 summer. He has also had a cooperate research projects with others with titles Automatic target Recognition (ATR), Electro-Optics/Infrared, and RF, and focused on the areas of analysis and development of Progressive Compression Algorithms, Visual Evaluation Tools for Geolocation, and Sensor Fusion Algorithm Development. His email address is 1uo@subr.edu.

SEDDIK M. DJOUADI received his Ph.D. degree from McGill University, his M.Sc. degree from University of Montreal, both in Montreal, his B.S. (with first class honors) from Ecole Nationale Polytechnique, Algiers, all in electrical engineering. He is currently a full professor in the Electrical Engineering and Computer Science Department at the University of Tennessee, Knoxville. He received the best paper award in the 1st Conference on Intelligent Systems and Automation 2008, the Ralph E. Powe Junior Faculty Enhancement Award in 2005, the Tibbet Award with AFS Inc. in 1999, and the American Control Conference Best Student Paper Certificate (best five in competition) in 1998. He has published over a hundred journal and conferences papers some of which were invited papers. His research interests include filtering and control of systems under communication constraints, modeling and control of wireless networks, control systems and applications to autonomous sensor platforms, electromechanical and mobile communication systems, in particular smart grid and power systems, control systems through communication links, networked control systems, and model reduction for aerodynamic feedback flow control. His email address is mdjouadi@utk.edu.

MOHAMMED M. OLAMA received the B.S. and M.S. (Hons.) degrees in electrical engineering from the University of Jordan, in 1998 and 2001, respectively, and the Ph.D. degree from the Electrical Engineering and Computer Science (EECS) Department, The University of Tennessee, Knoxville, TN, USA, in 2007. From 1999 to 2001, he served as a full-time Control Engineer with the National Electric Power Company, Amman, Jordan. He is currently a Research Scientist with the Computational Sciences and Engineering Division, Oak Ridge National Laboratory (ORNL), Oak Ridge, TN, USA. He has been with ORNL since 2007. He is also an Adjunct Associate Professor with the EECS Department, The University of Tennessee, Knoxville, TN, USA. He has over 150 technical publications, including articles in refereed journals, conference proceedings, book chapters, and technical reports; and numerous presentations at professional conferences and international symposia. His research interests include smart grid and smart buildings; smart grid communications and control; building-to-grid integration; cyberphysical systems; complex systems; wireless communications; 5G wireless networks; wireless sensor networks; wireless security; big data integration and analytics; healthcare data analytics and hazard detection; machine learning; artificial intelligence; statistical signal processing; and discrete-event simulation. Dr. Olama is a member of the Phi Kappa Phi Honor Society and a senior IEEE member. He received the Best Paper Awards in the IEEE International Symposium on Power Electronics for Distributed Generation Systems, in 2018, and the Mediterranean Conference on Intelligent Systems and Automation, in 2008. He was the recipient of the Significant Event Award at ORNL, in 2007, and the Best Mentor Award at ORNL, in 2019. His email address is olamahussemm@ornl.gov.

YICHEN ZHANG received the B.S. degree from Northwestern Polytechnical University, Xi'an, China, in 2010, M.S. degree from Xi'an Jiaotong University, Xi'an, China, in 2012, and Ph.D. degree in the Department of Electrical Engineering and Computer Science at the University of Tennessee, Knoxville, TN, USA, in 2018, all in Electrical Engineering. He is currently a postdoctoral appointee in the Energy Systems Division at Argonne National Laboratory. His research interests include power system dynamics, renewable energy, control systems and hybrid systems. His email address is yichen.zhang@anl.gov. 